\title{
Anticollinear magnetic order induced by impurities in the frustrated Heisenberg model of pnictides
}

\author{
Cédric Weber ${ }^{1,2, *}$ and Frédéric Mila ${ }^{3}$ \\ ${ }^{1}$ King's College London, Theory and Simulation of Condensed Matter (TSCM), The Strand, London WC2R 2LS, United Kingdom \\ ${ }^{2}$ Cavendish Laboratory, J. J. Thomson Avenue, Cambridge CB3 OHE, United Kingdom \\ ${ }^{3}$ Institute of Theoretical Physics, École Polytechnique Fédérale de Lausanne (EPFL), 1015 Lausanne, Switzerland
}

(Received 3 July 2012; published 29 November 2012)

\begin{abstract}
We present Monte Carlo simulations for a classical antiferromagnetic Heisenberg model with both nearest $\left(J_{1}\right)$ and next-nearest $\left(J_{2}\right)$ exchange couplings on the square lattice in the presence of nonmagnetic impurities. We show that the order-by-disorder entropy selection, associated with the Ising-like phase transition that appears for $J_{2} / J_{1}>1 / 2$ in the pure spin model, is quenched at low temperature due to the presence of nonmagnetic impurities. Evidence that a new competing order is stabilized around the impurities and in turn induces a reentrance phase transition is reported. Implications for local magnetic measurement of the parent compound of iron pnictides are briefly discussed.
\end{abstract}

DOI: 10.1103/PhysRevB.86.184432

PACS number(s): 75.10.Hk, 64.60.De

\section{INTRODUCTION}

Unconventional superconductivity occurs in the proximity of magnetically ordered states in many materials. ${ }^{1,2}$ Understanding the magnetic phase of the parent compound is an important step towards understanding the mechanism of superconductivity. Unlike the case of the cuprates, magnetism and its underlying electronic state in the iron pnictide superconductor $\mathrm{BaFe}_{2} \mathrm{As}_{2}{ }^{3}$ is still debated. Many low-energy probes such as transport, ${ }^{4}$ scanning tunneling microscopy, ${ }^{5}$ and angleresolved photoemission spectroscopy ${ }^{6}$ have measured strong in-plane anisotropy of the electronic states, but there is no consensus on its physical origin. It was suggested from first-principle calculations ${ }^{7}$ that the origin stems from orbital order, but the obtained anisotropy in the resistivity is opposite to the one found experimentally. ${ }^{8}$

A more likely scenario supported by recent neutron diffraction measurements ${ }^{9}$ is related to a spin density wave instability due to the presence of electron and hole pockets around $\mathbf{k}=(\pi, 0)$ and $\mathbf{k}=(0, \pi)$. The resulting magnetic order is of nematic type and can be seen as a helicoidal magnetic state with pitch vector $\mathbf{Q}=(0, \pi)$ or $\mathbf{Q}=(\pi, 0)$. Since the orbital character of the electron and hole pockets depends on the wave vector of the instability, ${ }^{10}$ the nematic order introduces in turn an orbital polarization.

To describe the low-energy magnetic properties of this system, it has been suggested early on that a local moment picture may become relevant in the presence of moderately large electronic correlations, ${ }^{11}$ leading to the Heisenberg model with both nearest $\left(J_{1}\right)$ and next-nearest $\left(J_{2}\right)$ exchange couplings defined by

$$
\hat{\mathcal{H}}=\sum_{\langle i, j\rangle} J_{1} \hat{\mathbf{S}}_{i} \cdot \hat{\mathbf{S}}_{j}+\sum_{\langle i, j\rangle\rangle} J_{2} \hat{\mathbf{S}}_{i} \cdot \hat{\mathbf{S}}_{j}
$$

In the collinear regime, both $J_{1}$ and $J_{2}$ are positive, and $2 J_{2}>$ $J_{1}{ }^{12}$ In this expression, $\hat{\mathbf{S}}_{i}$ are $\mathrm{O}(3)$ spins on a periodic square lattice with $N=L \times L$ sites. $\langle i, j\rangle$ and $\langle\langle i, j\rangle\rangle$ indicate the sum over nearest and next-nearest neighbors, respectively. ${ }^{13}$

The first attempt at fitting the experimental spin density wave excitation spectra with a Heisenberg model suggested that one should use very anisotropic values of $J_{1} \cdot{ }^{14}$ However, it was later shown that the fits of the experimental data included energy scales beyond $100 \mathrm{meV}$, which are not well described by magnon excitations. ${ }^{15} \mathrm{~A}$ more careful study, including the itinerant character of the electrons,${ }^{16}$ led to the conclusion that pnictides are indeed in the collinear regime with $(\mathbf{Q}=$ $(0, \pi),(\pi, 0))$ magnetic instabilities, a conclusion supported by first-principles calculations for selenium-based compounds $\left(\mathrm{KFe}_{2} \mathrm{Se}_{2}\right) .{ }^{17} \mathrm{We}$ also note that it was also recently argued that ${ }^{18}$ to get a proper description of magnetic interactions and spin fluctuations in ferropnictides, additional biquadratic interactions might be important.

In parallel, it has been suggested both experimentally ${ }^{19,20}$ and theoretically ${ }^{21}$ that impurities have a dramatic impact on the magnetic and superconducting properties. In particular, recent magnetic polarized $\mathrm{x}$-ray measurements suggest that a new type of magnetic order emerges due to the presence of magnetic impurities in $\mathrm{BaFe}_{2} \mathrm{As}_{2} .{ }^{22}$ Furthermore, periodic ordering of supercell structures of vacancies in $\mathrm{TlFe}_{1.6} \mathrm{Se}_{2}$ observed by electron microscopy was shown to induce a spin reorientation in these structures. ${ }^{23,24}$ All these results, together with results obtained a few years ago on a layered vanadium oxide ${ }^{25}$ call for an in-depth investigation of the effect of impurities in the frustrated Heisenberg model of Eq. (1).

In this paper, we address the question of the interplay between the frustration, induced by the exchange coupling, and the disorder induced by the imperfections of the crystallographic structure. Since density functional calculations, and quite generally quantum-based calculations, are limited to relatively small unit cells and cannot tackle the issue of large supercell structures, we limit our calculations to a frustrated classical model ${ }^{26}$ and carry out Monte Carlo calculations of the Heisenberg $J_{1}-J_{2}$ model in the presence of impurities using the same numerical approach as in Refs. 27 and 28 . We then extend the calculations to magnetic impurities.

\section{DISTORTION AROUND A SINGLE IMPURITY}

We first discuss the symmetry of the magnetic order for the case of a single impurity. In the absence of disorder and at zero temperature, the ground state has Néel order with magnetic vector $\mathbf{Q}=(\pi, \pi)$ for $J_{2} / J_{1}<0.5$, while for $J_{2} / J_{1}>0.5$ the ground state is continuously degenerate and can be described as two distinct antiferromagnetically ordered states on two 


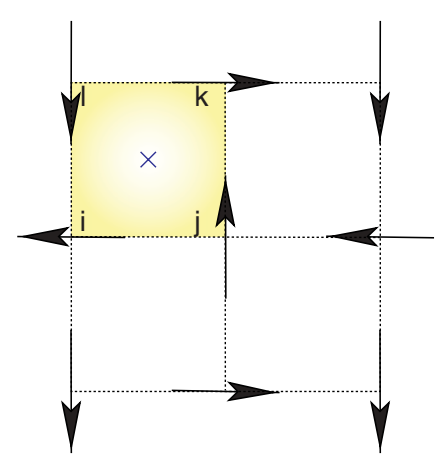

FIG. 1. (Color online) Sketch of anticollinear order. The letters $i, j, k$ and $l$ refer to the labeling of the sites of a plaquette used in the definition of the $Z_{2}$ and $M_{90}$ order parameters.

sublattices, with a relative angle $\theta$ between their magnetization axis. At finite temperature, the entropy selection reduces the $\mathrm{O}$ (3) symmetry of the ground state to $Z_{2}$ at finite temperature, selecting the states with antiferromagnetic spin correlations in one spatial direction and ferromagnetic correlations in the other $(\mathbf{Q}=(0, \pi),(\pi, 0))$. This is the so-called order by disorder entropic selection, and the associated discrete symmetry breaking drives a finite-temperature Ising-like phase transition. ${ }^{27,29}$

To characterize this transition, it is useful to construct, from the original spin variables $\hat{\mathbf{S}}_{i}$, an effective Ising variable on the dual lattice:

$$
M_{2}(x)=\left(\hat{\mathbf{S}}_{i}-\hat{\mathbf{S}}_{k}\right) \cdot\left(\hat{\mathbf{S}}_{j}-\hat{\mathbf{S}}_{l}\right),
$$

where $(i, j, k, l)$ are the corners with diagonal $(i, k)$ and $(j, l)$ of the plaquette centered at the site $x$ of the dual lattice (see Fig. 1), and we define its normalized counterpart as $Z_{2}(x)=$ $M_{2}(x) /\left|M_{2}(x)\right|$. In this way, the two collinear states with $\mathbf{Q}=$ $(\pi, 0)$ and $\mathbf{Q}=(0, \pi)$ can be distinguished by the value of the Ising variable, $Z_{2}(x)= \pm 1$

Our Monte Carlo calculations show that introducing a single impurity lifts the former continuous degeneracy and selects the state with $\theta=90^{\circ}$, as shown in Fig. 2(a) and in agreement with a prediction by Chris Henley ${ }^{30}$ who suggested the name (a)

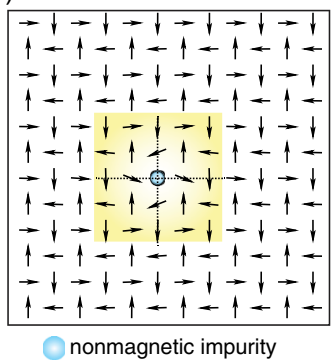

(b)

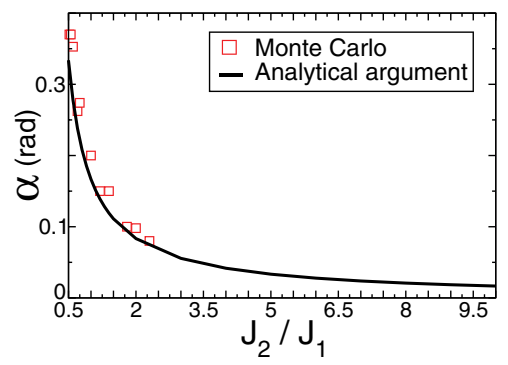

FIG. 2. (Color online) (a) Typical spin configuration obtained at $T=10^{-6} J_{1}$. The filled circle indicates the location of the impurity, and the rectangle highlights the region around the impurity where the spins deviate significantly from the $90^{\circ}$ ordered state for $J_{2} / J_{1}=$ 0.55. (b) Angle of the spins connected to the impurity with the horizontal axis as obtained from the Monte Carlo (squares) and with a simple variational criteria where only the spins connected to the impurity are tilted (line).

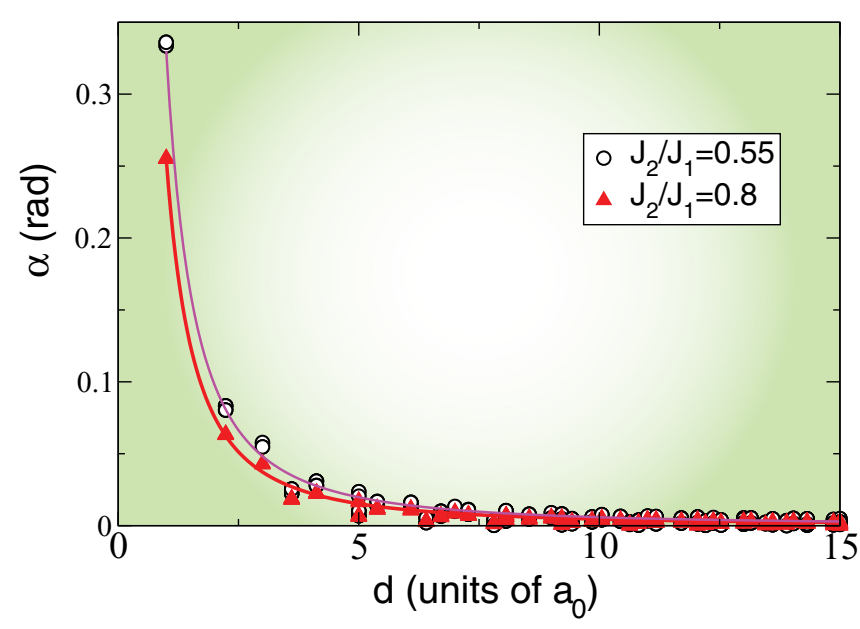

FIG. 3. (Color online) Distortion angle $\alpha$ as a function of the Manhattan distance $d$ from the impurity (in units of the lattice spacing $a_{0}$ ) obtained by Monte Carlo for $J_{2} / J_{1}=0.55$ (circles) and $J_{2} / J_{1}=$ 0.8 (triangles). Power-law fits to the data with exponent $\lambda=-2$ are shown for comparison (bold lines).

anticollinear to describe the state with $\theta=90^{\circ}$, as well as with linear spin-wave calculations on small clusters. ${ }^{31}$ The columnar states were also suggested as a stable phase of ferropnictides ${ }^{16}$ in the itinerant picture. The anticollinear state can be characterized similarly to the Ising order by the norm of a pseudovector:

$$
M_{90}(x)=\left|\left(\hat{\mathbf{S}}_{i}-\hat{\mathbf{S}}_{k}\right) \wedge\left(\hat{\mathbf{S}}_{j}-\hat{\mathbf{S}}_{l}\right)\right| .
$$

This selection is induced by a local energy optimization around the impurity site. Indeed, the four spins on the impurity's nearest-neighbor sites slightly rotate to acquire a small common ferromagnetic component in order to optimize locally the energy with their own neighbors once an impurity is introduced into the $90^{\circ}$ state. We show in Fig. 2(b) the resulting deviation of the spins connected to the impurity from the bulk $90^{\circ}$ magnetic state. This energetic optimization spreads further through the lattice and affects the spins belonging to the same sublattice, while the spins on the other sublattice are marginally affected. We show in Fig. 3 the obtained deviation of the spins from the bulk $90^{\circ}$ magnetic state as a function of the distance from the impurity. Clearly the distortion is large close to the impurity, as confirmed by fits to the data, which are consistent with a power-law decay with an exponent $\lambda \approx-2$.

It is noteworthy that treating the distortion angle $\alpha$ as a simple variational parameter and neglecting the distortion of the spins which are not nearest neighbors of the impurity lead to a very good estimate of the Monte Carlo result. The good agreement between the two methods confirms that the energy optimization around a single impurity is essentially local in space, and thus the selection of the $90^{\circ}$ magnetic state is driven by a local energetic optimization process, in contrast to the entropic order-by-disorder selection of the $\mathbf{Q}=(0, \pi),(\pi, 0)$ states in the clean system.

Let us now turn to the discussion of the single-impurity problem at small but finite temperature. The $90^{\circ}$ spin order around a single impurity is shown in Figs. 5(a) and 5(c). Since the $90^{\circ}$ order does not break discrete symmetry, in contrast to the Ising symmetry broken by the $Z_{2}$ Ising 


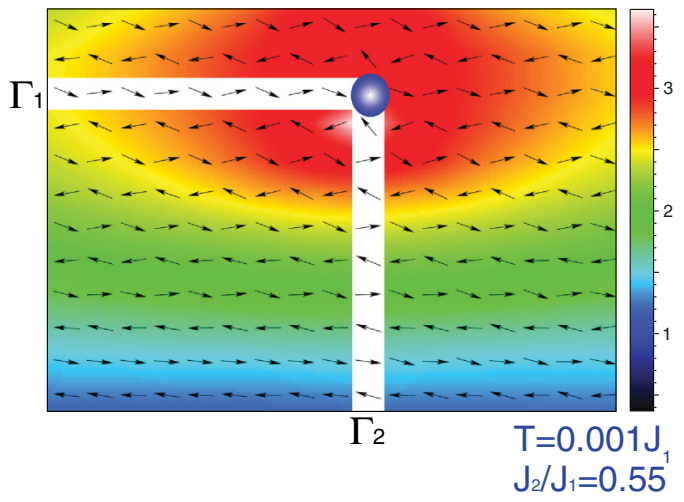

FIG. 4. (Color online) Color plot of $M_{90}(x)$ at $T=10^{-3} J_{1}$ (blue and red are respectively the minimum and maximum). This calculation is done for $J_{2} / J_{1}=0.55$.

parameter, it is not a stable thermodynamic phase. At finite temperature, the anticollinear order characterized by Eq. (3) has a finite correlation length. Interestingly, the anticollinear order remains strong within a finite region around the impurity (see Fig. 4), and outside this region the collinear $(0, \pi),(\pi, 0)$ states are recovered.

Thus, the $90^{\circ}$ order does not develop long-range correlations but is rather stabilized around the impurity within a finite region: At finite temperature, there is a competition in the free energy $F=E-T S$ between, on the one hand, the local energy optimization in the vicinity of the impurity which favors the $90^{\circ}$ spin order, and on the other hand the entropy selection which favors the Ising $\mathbf{Q}=(0, \pi),(\pi, 0)$ states. Notwithstanding that the $90^{\circ}$ state is energetically favored and is stabilized at short distances from the impurity, we indeed find that thermal fluctuations screen the impurity at long distances, and that the system recovers the entropically selected Ising states far from the impurity. Since the entropic mechanism is temperature dependent, so is the size of the $90^{\circ}$ cluster: It increases when the temperature decreases, as can be seen from Fig. 5(a) obtained at $T=10^{-3} J_{1}$ and Fig. 5(c) obtained at a smaller temperature $T=10^{-4} J_{1}$. In addition, we note that the shape of the $90^{\circ}$ cluster is highly asymmetric. To understand this point, we measured the Ising order parameter $M_{2}$ in the same calculation [see Figs. 5(b) and 5(d)]. As expected, we observe a concomitant reduction of the Ising order in the region where the $90^{\circ}$ order is large, but more remarkably, we find that the shape of the $90^{\circ}$ cluster correlates with the Ising order: The cluster is an ellipsoid with a large axis $\Gamma_{1}$ in the direction along which the spins are parallel and a small axis $\Gamma_{2}$ in the direction along which the spins are antiparallel. Furthermore, the spins along $\Gamma_{1}\left(\Gamma_{2}\right)$, highlighted by the white stripes in Fig. 4, correspond to the ferromagnetically (antiferromagnetically) aligned spins of the collinear $(0, \pi),(\pi, 0)$ states. Hence, due to the antiferromagnetic $J_{1}$ coupling, the deviations from the pure Ising state are energetically favorable along $\Gamma_{1}$, and costly along $\Gamma_{2}$, which explains why the screening of the impurity is more effective in one direction than the other.

\section{FINITE CONCENTRATION}

Let us now extend the discussion to the averaged physical observables with a finite concentration of impurities $\delta$ [see
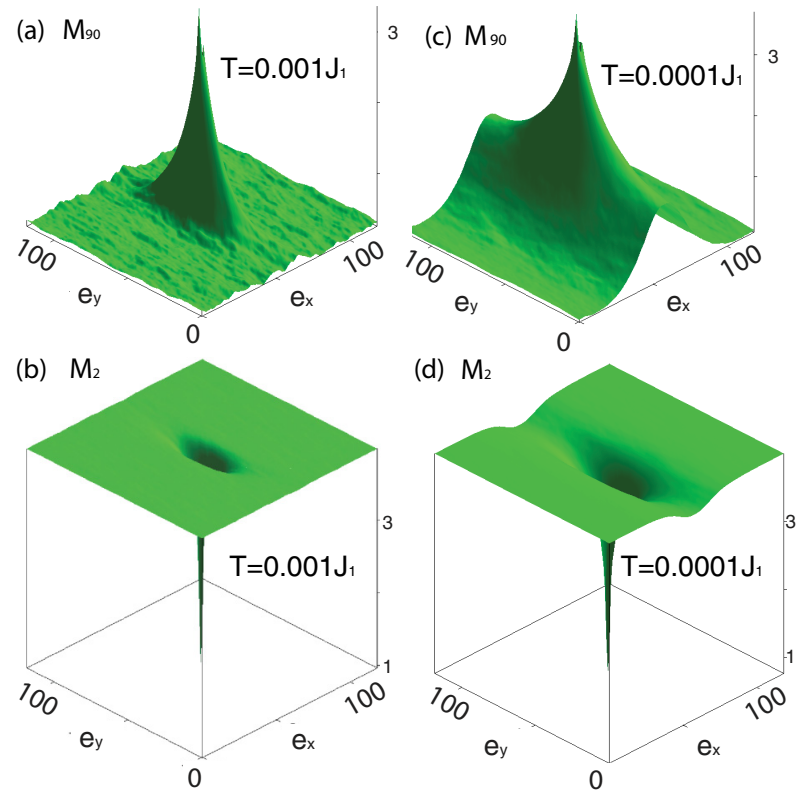

FIG. 5. (Color online) Spatially resolved order parameter (a) for the $90^{\circ}$ spin order $\left[M_{90}(x)\right]$ and $(b)$ for the Ising order $\left[M_{2}(x)\right]$ obtained at $T=10^{-3} J_{1}$. The respective order parameters evaluated at a lower temperature $T=10^{-4} J_{1}$ are shown in (c) and (d). In all the calculations above the cluster contains $L \times L=120 \times 120$ sites and the impurity is located at the center of the cluster at $(x, y)=(60,60)$.

Fig. 6(a)]. We carried out Monte Carlo calculations for a $L \times L=40 \times 40$ cluster with different magnetic impurities dilutions $\delta$, and we averaged the physical observables over 32 random configurations of impurities. We find for small concentration $\delta=0.125 \%$ only a weak effect on the Ising order. In particular, we observe the Ising-like crossover at $T \approx 0.2 J_{1}$, as shown by the sharp drop of the order parameter at this temperature, and a very small dip in the $Z_{2}$ order parameter at $T \approx 0.005 J_{1}$. The phase diagram of Fig. 6(b) is consistent with earlier numerical calculations by Henley and Prakash. ${ }^{30}$ In particular, the re-entrance of the Ising order, reported for the $X Y$ version model in that reference, is also present in the Heisenberg model [see Fig. 6(a)].

We note that transitions belonging to the Ising universality class ( $v=1$ and the dimension $d=2$ ) do not satisfy the wellknown Harris criteria, ${ }^{32}$ which assess that phase transitions with $v d>2$ are unaffected by the disorder. So it is not obvious a priori whether the transition into the collinear phase is a true phase transition or a crossover. While numerical studies have shown that the simple 2D Ising model is weakly affected by disorder, ${ }^{33}$ it has been argued in Ref. 30 for the $X Y$ model that the disorder induced by vacancies does not induce a genuine order in 2D. A formal proof would require a detailed finite size scaling analysis and goes beyond the scope of this work.

At larger concentrations $0.125 \%<\delta<11 \%$, we find a steady decrease of the Ising order at small temperatures, as highlighted by the dashed lines in Fig. 6(a). For instance, at $\delta=$ $2 \%$ we observe a quench of the Ising order for $T<0.025 J_{1}$, and the entropic selection kicks in for $0.025<T<0.19$ 


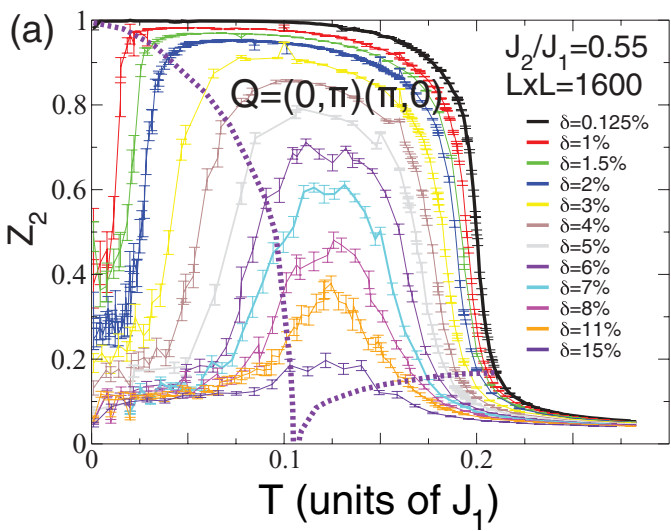

(b)

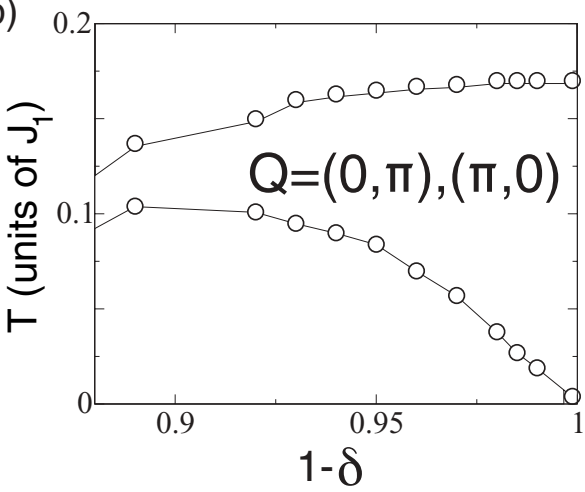

FIG. 6. (Color online) (a) Temperature dependence of the spatially averaged Ising order parameter $Z_{2}$ for various impurity concentrations $\delta$ obtained for a $L \times L=40 \times 40$ lattice. The Ising order is suppressed at low temperature by the presence of impurities, and at large temperature by strong thermal fluctuations. The dashed lines are guide to the eyes to track the Ising crossovers at low and high temperatures. (b) Corresponding phase diagram in impurity density $\delta$ and temperature T. Calculations above were averaged over 32 random configurations of disorder.

where we obtain the Ising ordered phase, and finally at higher temperatures $T>0.19$ the system is a paramagnet. So we are in the presence of a reentrance phase transition. We note that the impurities mainly affect the Ising order at low temperatures, and the Ising-like transition near $T \approx 0.2 J_{1}$ is moderately affected by impurities at small and moderate dilutions. Beyond a critical dilution $\delta_{c} \approx 20 \%$, we do not observe the presence of the collinear or anticollinear states. Indeed, in two dimensions and for an impurity dilution $\delta_{c}=$ $1 / 9$, there is one impurity on average connected to every spin of the lattice, so that the local distortions and subsequent local energy optimizations start to prevail over both the Ising phase and the $90^{\circ}$ local spin order. The phase diagram is summarized in Fig. 6(b). Note that the reentrant behavior of the Ising phase agrees with the prediction of Ref. 30. However, the rest of the phase diagram of Ref. 30 cannot be compared to the present results. Indeed, in the case of the $X Y$ model studied in Ref. 30, the local chirality of the anticollinear order defines naturally an Ising variable, and the anticollinear phase must be separated from the paramagnetic phase by a phase transition. By contrast, here the low-temperature phase below the Ising phase can be smoothly connected to the paramagnetic phase. Nonetheless, the long-range order for the vector chirality at

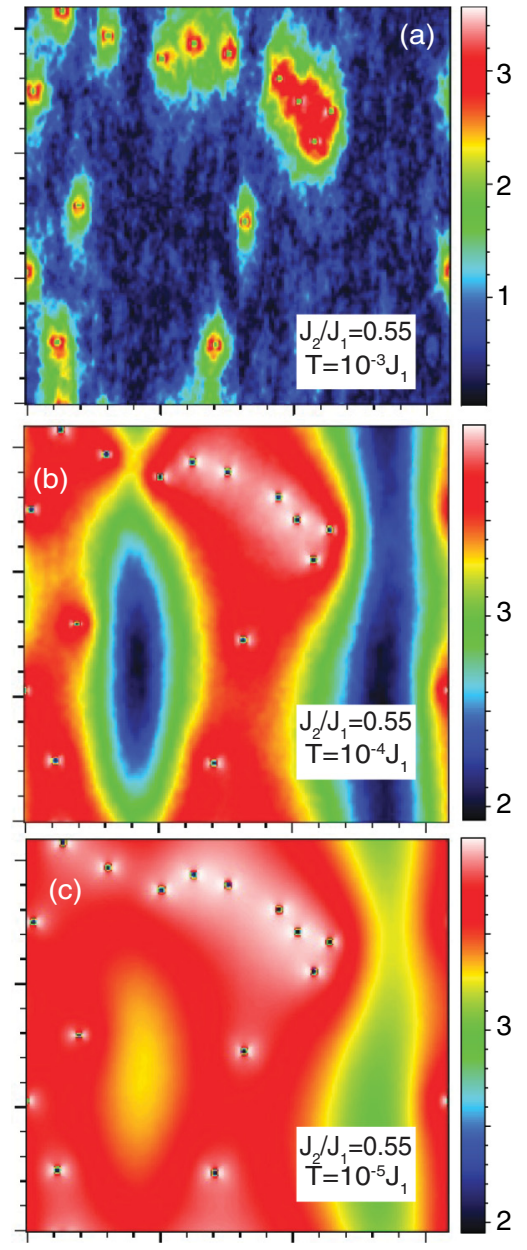

FIG. 7. (Color online) Real-space map of $M_{90}$ obtained for a given configuration of 15 nonmagnetic impurities in a $L \times L=$ $160 \times 160$ lattice $(\delta=0.06 \%)$ for $J_{2} / J_{1}=0.55$ at (a) $T=10^{-3} J_{1}$, (b) $T=10^{-4} J_{1}$. The colors range from black (minimum) to red (maximum).

finite temperature for Heisenberg spins could accommodate an order parameter built upon vortices, ${ }^{34}$ and we cannot rule out a true phase transition at small but finite temperature. To study this crossover would require studying larger dilutions and performing a systematic finite size scalings, which goes beyond the scope of the present work.

The reentrance phenomena observed in Fig. 6(a) can be explained at small impurity concentration on the basis of the single-impurity results at finite temperature. To see this, we now consider a given configuration of impurities for a small but finite impurity dilution of $\delta=0.06 \%$ at $T=10^{-3}, 10^{-4}, 10^{-5} J_{1}$ and $J_{2} / J_{1}=0.55$ [see respectively Figs. 7(a), 7(b), and 7(c)]. The $90^{\circ}$ order stays localized around the impurities at high temperature [Fig. 7(a)], but forms superstructures connecting the impurities [Fig. 7(b)] upon lowering the temperature until it finally spreads through the whole lattice [Fig. 7(c)]. This process is very similar to a percolation transition, and can be captured within a very simple argument: The anticollinear order spreads through the whole system when the size of the $90^{\circ}$ clusters is of the order of the mean distance $\lambda$ between the impurities. More precisely, since the $90^{\circ}$ cluster is an ellipsoid, we obtain a lower and upper 

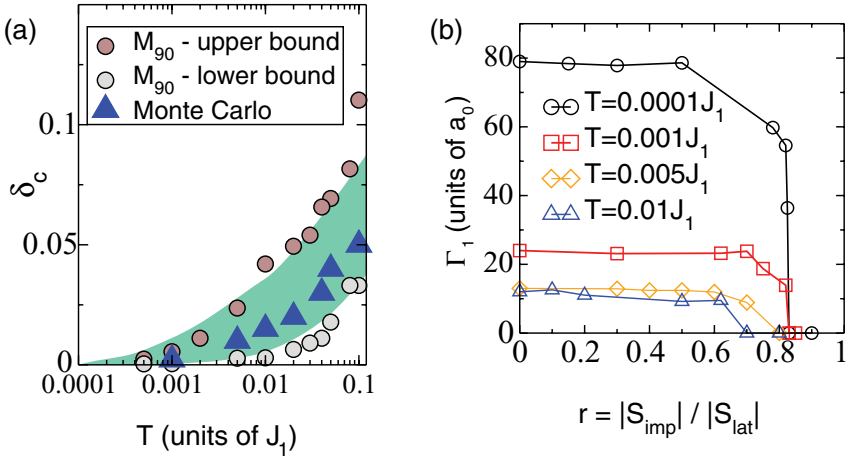

FIG. 8. (Color online) (a) Critical dilution $\delta_{c}$ as a function of temperature obtained from the Monte Carlo data of Fig. 6(a) (triangles), and by a simple criteria comparing the length of the short (dark gray circles) and long (light gray circles) ellipsoidal axis of the $90^{\circ}$ order cluster (see discussion in the text). (b) $\Gamma_{1}$ (the long axis of the $90^{\circ}$ order ellipsoid) in units of $a_{0}$ as a function of $r=S_{\text {imp }} / S_{\text {lat }}$, where $S_{\text {imp }}$ is the spin of the magnetic impurity, and $S_{\text {lat }}$ is the spin of the correlated element of the compound.

bound on the critical dilution, by comparing the long $\left(\Gamma_{1}\right)$ and short $\left(\Gamma_{2}\right)$ axis respectively to the mean impurity-impurity distance. We compare in Fig. 8(a) the critical dilution obtained from the Monte Carlo data of Fig. 6(a) with the critical dilutions obtained by this simple argument. We find that this argument provides a reliable estimate of the critical dilution. This confirms that the reentrance of the Ising order can be accounted for by comparing length scales associated with the competition between Ising and $90^{\circ}$ order on one hand and with the impurity concentration on the other hand. Interestingly, this is reminiscent of a recently reported study where the authors obtained a reentrance transition in a discrete spin model that describes a completely different system, namely hydrogen-bond physics for water layers. ${ }^{35}$

\section{EXTENSION TO MAGNETIC IMPURITIES}

We generalized our calculations to magnetic impurities with nonzero spin [see Fig. 8(b)]. Remarkably, we find that the $90^{\circ}$ order cluster around a single impurity is not significantly affected by the spin of the impurity $S_{\text {imp }}$, as long as the ratio of the spin of the impurity to the magnetic element of the compound $r=S_{\text {imp }} / S_{\text {lat }}$ remains smaller than $\approx 0.6$. This suggests that the reentrance phase transition does not strictly require nonmagnetic impurities but could also be present for instance in the case of $\mathrm{Ni}$ impurities in $\mathrm{BaFe}_{2} \mathrm{As}_{2}$, where the spin of $\mathrm{Ni}$ is $\approx 40 \%$ of the spin of Fe. ${ }^{36}$ So it will be very interesting to see whether the order proposed in this paper can lead to an alternative interpretation of the NMR results in $\mathrm{BaFe}_{2} \mathrm{As}_{2}$ and maybe help clarify the origin of the line shapes in $\mathrm{Ni}$ - and $\mathrm{Zn}$-doped samples. ${ }^{36}$ We note that the magnetic distortion around a single impurity was also studied in the context of magnetic frustration in the triangular lattice. ${ }^{37}$ For magnetic impurities in noncollinear magnets, the authors of Ref. 37 find that there is only a partial screening of the impurity magnetic moment, leading to a fractional Curie response at low temperatures in the $2 \mathrm{D}$ case. How this compares to the frustrated Heisenberg model on the square lattice will be interesting to be clarified in future work.

\section{DEPENDENCE ON $J_{2} / J_{1}$}

Finally, we extended the calculations to other values of $J_{2} / J_{1}$, in order to assess the range of parameters where our theory is valid. In order to estimate the range of $J_{2} / J_{1}$ parameters where the anticollinear order is stabilized, we compute the energy gain obtained by the distortion $\alpha$ near the impurity site at zero temperature for the case of a single impurity. Since the distortion $\alpha$ is mostly local, we neglect the distortion for the spins which are not nearest neighbors of the impurity. This can be obtained straightforwardly:

$$
\begin{aligned}
E(\lambda= & \left.J_{2} / J_{1}, \alpha\right) / J_{1}=4 \cos (\pi / 2-\alpha)+8 \lambda \cos (\pi-\alpha) \\
& +4 \lambda \cos (\pi-2 \alpha)+8 \cos (\pi / 2+\alpha)+12 \lambda .
\end{aligned}
$$

As shown in Fig. 9(a), the energy gain at zero temperature decreases monotonically when $J_{2} / J_{1}$ increases, and becomes small for $J_{2} / J_{1}>1.5$. The latter is confirmed by Monte Carlo
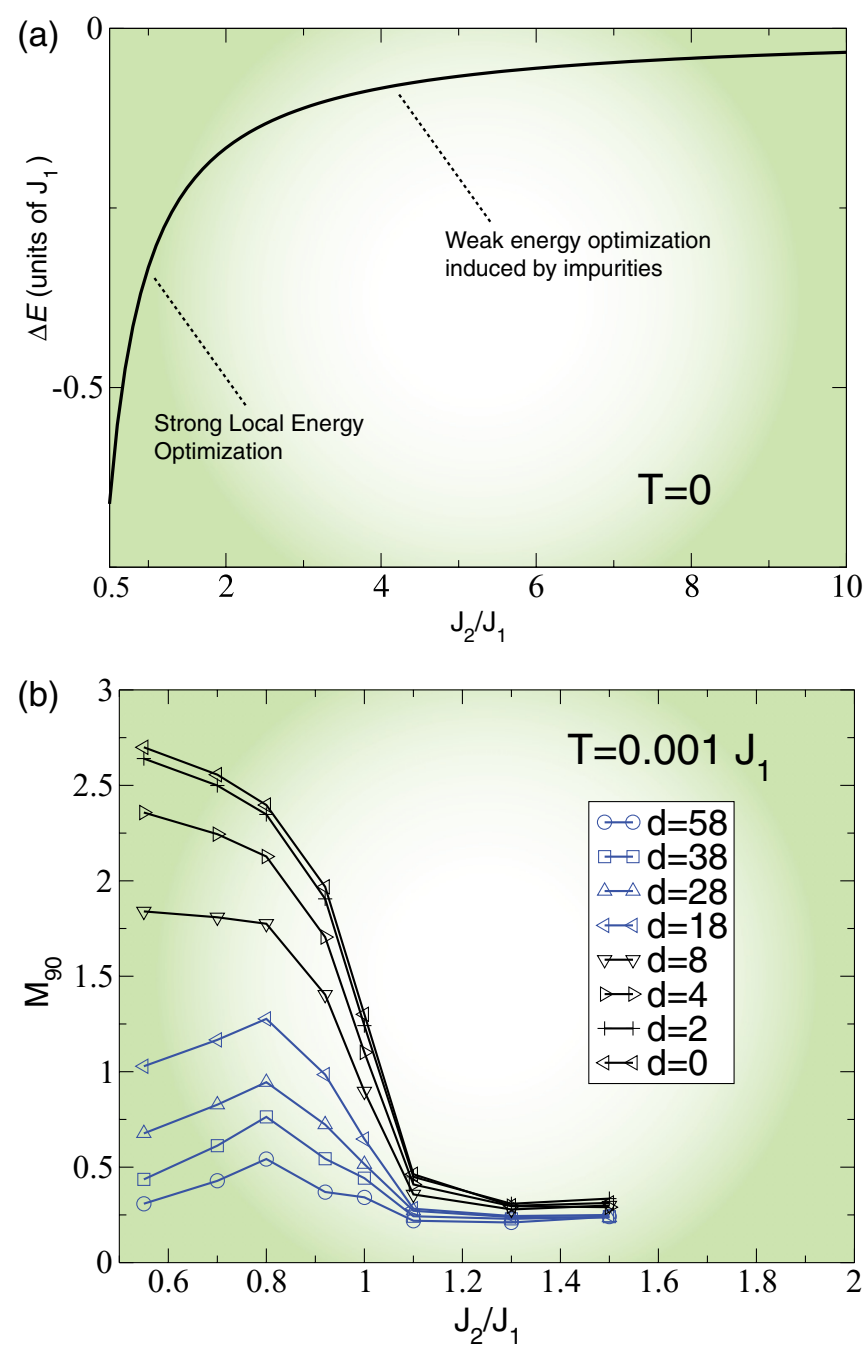

FIG. 9. (Color online) (a) Energy gain $E(\alpha)-E(\alpha=0)$ at zero temperature as a function of $J_{2} / J_{1}$ computed with a simple variational argument where only the nearest-neighbor sites of the impurity distort from the $90^{\circ}$ order. (b) Anticollinear order obtained by Monte Carlo at finite temperature $T=0.001 J_{1}$ and spatially resolved at a given distance $d$ from the impurity as a function of $J_{2} / J_{1}$. 


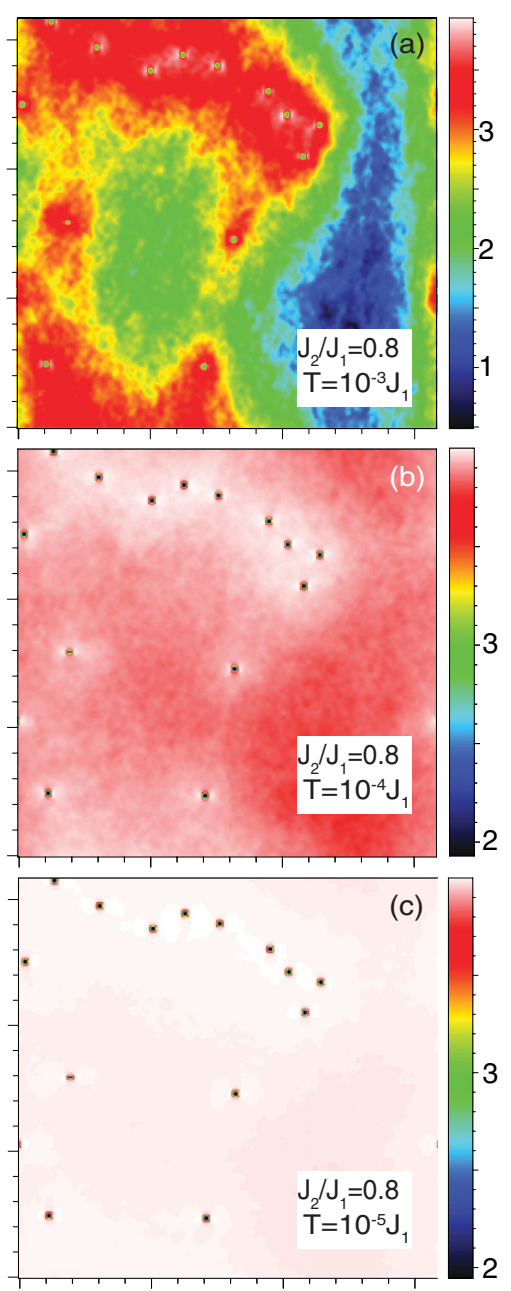

FIG. 10. (Color online) Real-space map of $M_{90}$ obtained for a given configuration of 15 nonmagnetic impurities in a $L \times L=$ $160 \times 160$ lattice $(\delta=0.06 \%)$ for $J_{2} / J_{1}=0.8$ at (a) $T=10^{-3} J_{1}$, (b) $T=10^{-4} J_{1}$, and (c) $T=10^{-5} J_{1}$. The colors range from black (minimum) to red (maximum).

calculations at finite temperature $T=0.001 J_{1}$ [see Fig. 9(b)], where we resolve spatially the anticollinear order at a fixed distance from the impurity, and compute how it evolves when $J_{2} / J_{1}$ increases. Indeed, we find that for all distances from the impurity, the anticollinear order is suppressed for $J_{2} / J_{1}>1.1$.

It is noteworthy that the anticollinear order is strikingly increasing with $J_{2} / J_{1}$ at large distances from the impurity (18 lattice spacings and more) and for $J_{2} / J_{1}<0.8$. We attribute this effect to the fact that the collinear order is competing with the antiferromagnetic Néel order near $J_{2} / J_{1}=$
0.5 , which leads to large thermal fluctuations in the critical regime. Indeed, the latter was shown to suppress the Ising phase transition and the critical temperature $T_{c}$ goes to zero at $J_{2} / J_{1}=0.5 .^{27}$ The larger fluctuations, which give a small $T_{c}$ near $J_{2} / J_{1}=0.5$, are hence expected to also reduce in turn the anticollinear order far from the impurity, since the local energy optimization is screened faster by large entropic contributions.

This is also confirmed by calculations done for a finite concentrations of impurities $\delta=0.06 \%$ (see Fig. 10). We find that for $J_{2} / J_{1}=0.8$ [see respectively Figs. 10(a), 10(b), and 10(c)] the anticollinear order parameter is larger at all temperatures $T=10^{-3}, 10^{-4}, 10^{-5} J_{1}$ than for the corresponding temperatures at $J_{2} / J_{1}=0.55$.

\section{CONCLUSION}

In conclusion, we have carried out a systematic study of the effect of nonmagnetic impurities in a frustrated Heisenberg model. We reported that for $J_{2} / J_{1}>0.5$ the continuous degeneracy of the ground state induced by the frustration is lifted due to a local optimization in the vicinity of a single impurity. The energy gain favors anticollinear order, which consists of bipartite lattices supporting Néel states entangled with a $90^{\circ}$ angle. This order, energetically favored, competes with the Ising order, entropically favored, and at long distance we find that the impurity is screened by thermal fluctuations. This results in a rich phase diagram with a reentrant collinear phase upon increasing the temperature for a finite dilution.

Moreover, we have shown that the structure around the impurity locally departs from the purely anticollinear order. This effect is large when $J_{2} / J_{1}$ is close to $1 / 2$, as in the pnictides, and should be detectable by local probes such as NMR. It would also be interesting to investigate the implications of these results in the context of vanadates. ${ }^{25}$ An extension of these calculations to the itinerant picture will be interesting in the near future, by using variational Monte Carlo based methods ${ }^{38,39}$ in the presence of noncollinear magnetism. ${ }^{40}$

\section{ACKNOWLEDGMENTS}

We would like to thank N. Curro for very useful explanations about the NMR results of Ref. 36, and F. Becca and A. Läuchli for interesting discussions at an early stage of the project. One of us (C.W.) acknowledges very interesting discussions with D. Inosov. We are especially indebted to I. Eremin, C. Henley, and A. Läuchli for quite insightful suggestions following their critical reading of the manuscript. This work was supported by the Swiss National Fund and by MaNEP. *cedric.weber@kcl.ac.uk

${ }^{1}$ Y. Takabayashi, A. Y. Ganin, P. Jeglic, D. Arcon, T. Takano, Y. Iwasa, Y. Ohishi, M. Takata, N. Takeshita, K. Prassides, and M. J. Rosseinsky, Science 323, 1585 (2009).

${ }^{2}$ T. Park, F. Ronning, H. Q. Yuan, M. B. Salamon, R. Movshovich, J. L. Sarrao, and J. D. Thompson, Nature (London) 440, 65 (2006). ${ }^{3}$ Y. Kamihara, T. Watanabe, M. Hirano, and H. Hosono, J. Am. Chem. Soc. 130, 3296 (2008).
${ }^{4}$ J.-H. Chu, J. G. Analytis, K. De Greve, P. L. McMahon, Z. Islam, Y. Yamamoto, and I. R. Fisher, Science 329, 824 (2010).

${ }^{5}$ T.-M. Chuang, M. P. Allan, J. Lee, Y. Xie, N. Ni, S. L. Bud'ko, G. S. Boebinger, P. C. Canfield, and J. C. Davis, Science 327, 181 (2010).

${ }^{6}$ P. Richard, K. Nakayama, T. Sato, M. Neupane, Y.-M. Xu, J. H. Bowen, G. F. Chen, J. L. Luo, N. L. Wang, X. Dai, Z. Fang, H. Ding, and T. Takahashi, Phys. Rev. Lett. 104, 137001 (2010). 
${ }^{7}$ W. Lv, F. Krüger, and P. Phillips, Phys. Rev. B 82, 045125 (2010). ${ }^{8}$ B. Valenzuela, E. Bascones, and M. J. Calderón, Phys. Rev. Lett. 105, 207202 (2010).

${ }^{9}$ D. K. Pratt, M. G. Kim, A. Kreyssig, Y. B. Lee, G. S. Tucker, A. Thaler, W. Tian, J. L. Zarestky, S. L. Bud'ko, P. C. Canfield, B. N. Harmon, A. I. Goldman, and R. J. McQueeney, Phys. Rev. Lett. 106, 257001 (2011).

${ }^{10}$ R. M. Fernandes, E. Abrahams, and J. Schmalian, Phys. Rev. Lett. 107, 217002 (2011); J. Knolle, I. Eremin, A. Akbari, and R. Moessner, ibid. 104, 257001 (2010).

${ }^{11}$ Q. Si and E. Abrahams, Phys. Rev. Lett. 101, 076401 (2008).

${ }^{12}$ P. Chandra and B. Doucot, Phys. Rev. B 38, 9335 (1988).

${ }^{13} J_{1}$ sets the energy scale, and in our work $J_{2} / J_{1}=0.55$ is used when not specified otherwise, and both $J_{1}>0$ and $J_{2}>0$.

${ }^{14}$ J. Zhao, D. T. Adroja, D.-X. Yao, R. Bewley, S. Li, X. F. Wang, G. Wu, X. H. Chen, J. Hu, and P. Dai, Nat. Phys. 5, 555 (2009).

${ }^{15}$ R. A. Ewings, T. G. Perring, J. Gillett, S. D. Das, S. E. Sebastian, A. E. Taylor, T. Guidi, and A. T. Boothroyd, Phys. Rev. B 83, 214519 (2011).

${ }^{16}$ I. Eremin and A. V. Chubukov, Phys. Rev. B 81, 024511 (2010).

${ }^{17}$ C. Cao and J. Dai, Chin. Phys. Lett. 28, 057402 (2011).

${ }^{18}$ A. L. Wysocki, K. D. Belashchenko, and V. P. Antropov, Nat. Phys. 7, 485 (2011)

${ }^{19}$ S. Mukhopadhyay, S. Oh, A. M. Mounce, M. Lee, W. P. Halperin, N. Ni, S. L. Bud'ko, P. C. Canfield, A. P. Reyes, and P. L. Kuhns, New J. Phys. 11, 055002 (2009).

${ }^{20}$ P. Bonfà, P. Carretta, S. Sanna, G. Lamura, G. Prando, A. Martinelli, A. Palenzona, M. Tropeano, M. Putti, and R. De Renzi, Phys. Rev. B 85, 054518 (2012).

${ }^{21}$ C.-C. Chen, R. Applegate, B. Moritz, T. P. Devereaux, and R. R. P. Singh, New J. Phys. 13, 043025 (2011).

${ }^{22}$ M. P. M. Dean, M. G. Kim, A. Kreyssig, J. W. Kim, X. Liu, P. J. Ryan, A. Thaler, S. L. Bud'ko, W. Strassheim, P. C. Canfield, J. P. Hill, and A. I. Goldman, Phys. Rev. B 85, 140514 (2012).
${ }^{23}$ A. F. May, M. A. McGuire, H. Cao, I. Sergueev, C. Cantoni, B. C. Chakoumakos, D. S. Parker, and B. C. Sales, arXiv:1207.1318.

${ }^{24}$ R. Yu, P. Goswami, and Q. Si, Phys. Rev. B 84, 094451 (2011).

${ }^{25}$ N. Papinutto, P. Carretta, S. Gonthier, and P. Millet, Phys. Rev. B 71, 174425 (2005).

${ }^{26}$ G. Franzese, V. Cataudella, S. E. Korshunov, and R. Fazio, Phys. Rev. B 62, R9287 (2000).

${ }^{27}$ C. Weber, L. Capriotti, G. Misguich, F. Becca, M. Elhajal, and F. Mila, Phys. Rev. Lett. 91, 177202 (2003).

${ }^{28}$ C. Weber, F. Becca, and F. Mila, Phys. Rev. B 72, 024449 (2005).

${ }^{29}$ P. Chandra, P. Coleman, and A. Larkin, Phys. Rev. Lett. 64, 88 (1990).

${ }^{30}$ C. L. Henley, Phys. Rev. Lett. 62, 2056 (1989); J. Appl. Phys. 61, 3962 (1987); C. Henley and S. Prakash, J. Phys. Colloques 49, C8 (1988).

${ }^{31}$ B. Xu, C. Fang, W. Liu, and J. Hu, arXiv:1104.1848.

${ }^{32}$ A. B. Harris, J. Phys. C 7, 1671 (1974).

${ }^{33}$ F. Szalma and F. Igloi, J. Stat. Phys. 95, 763 (1999).

${ }^{34}$ H. Kawamura, J. Phys. Soc. Jpn. 55, 2157 (1986).

${ }^{35}$ E. G. Strekalova, M. G. Mazza, H. E. Stanley, and G. Franzese, Phys. Rev. Lett. 106, 145701 (2011).

${ }^{36}$ A. P. Dioguardi, N. Roberts-Warren, A. C. Shockley, S. L. Bud'ko, N. Ni, P. C. Canfield, and N. J. Curro, Phys. Rev. B 82, 140411 (2010).

${ }^{37}$ A. Wollny, L. Fritz, and M. Vojta, Phys. Rev. Lett. 107, 137204 (2011).

${ }^{38}$ C. Weber, A. Läuchli, F. Mila, and T. Giamarchi, Phys. Rev. Lett. 102, 017005 (2009).

${ }^{39}$ C. Weber, D. Poilblanc, S. Capponi, F. Mila, and C. Jaudet, Phys. Rev. B 74, 104506 (2006).

${ }^{40}$ C. Weber, A. Läuchli, F. Mila, and T. Giamarchi, Phys. Rev. B 73, 014519 (2006). 